\title{
Human Leukocyte Antigen-DR Isotype Expression in Monocytes and T Cells Interferon-Gamma Release Assay in Septic Patients and Correlation With Clinical Outcome
}

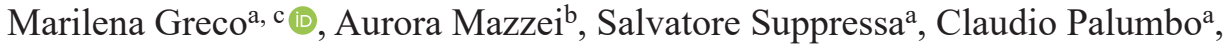 \\ Tiziano Verri ${ }^{\mathrm{b}}$, Giambattista Lobreglio ${ }^{\mathrm{a}}$
}

\begin{abstract}
Background: Sepsis is a life-threatening dysregulated host response to infection responsible of multiple organs dysfunction (Sepsis-3 International Consensus Definition), during which clinical outcome is a balance between inflammation and immune suppression. Monocytes and lymphocytes may play an important role in immune paralysis, and their impaired functional activity can decrease overall immune system efficiency. We evaluated sepsis-induced changes in monocytes human leukocyte antigen-DR isotype (HLA-DR) expression and $\mathrm{T}$ cell capacity of interferon (IFN)- $\gamma$ production in relation with patient's clinical outcome.
\end{abstract}

Methods: Analysis of HLA-DR expression on blood monocytes ( $\mathrm{mH}-$ LA-DR) was performed in 55 patients with high procalcitonin (hPCT, > $0.5 \mathrm{ng} / \mathrm{mL}$, ) and suspected/confirmed sepsis, and 20 controls. HLA-DR absolute quantification and IFN- $\gamma$ release assay were monitored in 16 septic patients for 4 weeks following sepsis confirmation.

Results: Cytofluorimetric analysis revealed a significant decrease of mHLA-DR percentage in septic patients with adverse outcome compared to patients with better clinical outcome ( $88.4 \%$ vs. $98.6 \%$ with $\mathrm{P}<0.05)$, in combination with a significant decrease of absolute number of HLA-DR molecules per monocyte $(\mathrm{P}<0.05$, starting at 1 week of follow-up). Lymphocytes stimulation with phytohemagglutinin (PHA), Staphylococcus aureus (S. aureus) and Candida albicans (C. albicans) showed a severe declining of IFN- $\gamma$ release related to fatal clinical outcome of patients.

Manuscript submitted March 9, 2021, accepted March 20, 2021

Published online May 25, 2021

${ }^{a}$ Clinical Pathology and Microbiology, Vito Fazzi General Hospital ASL-Lecce, Lecce, Italy

${ }^{b}$ Laboratory of Applied Physiology, Department of Biological and Environmental Sciences and Technologies (DeBEST), University of Salento, Lecce, Italy

${ }^{\mathrm{c} C}$ Corresponding Author: Marilena Greco, Clinical Pathology and Microbiology, Vito Fazzi General Hospital ASL-Lecce, 73100 Piazza Muratore, Lecce, Italy. Email: grecomarilena@gmail.com

doi: https://doi.org/10.14740/jocmr4474
Conclusions: This immunologic anergy of innate and adaptative immunity showed an early immune paralysis during sepsis which appears correlated with the impairment of clinical outcome.

Keywords: Immune paralysis; Sepsis; HLA-DR; Interferon- $\gamma$

\section{Introduction}

The immune response is an essential defense mechanism against infection and tissue injury through both inflammation and immune suppression [1], a process involving both innate and adaptative immunity. After the first phase characterized by inflammation and "cytokine storm", during which blood monocytes release high levels of pro-inflammatory cytokines (e.g., tumor necrosis factor (TNF)- $\alpha$ and interleukin (IL)-1) [2], the following stage of adaptative immune tolerance counteracts host's initial inflammatory response leading to the resolution of the infective process. This second phase could establish systemic state of immune paralysis, in which patients are characterized by persistent inflammation, neutrophilia, lymphopenia and susceptibility to secondary infections due to a dysregulation of innate and adaptive immunity [3].

Circulating monocytes play an important role in the defense of organism, cross-link innate and adaptive immunity and are involved in pathogenesis of several inflammatory diseases. Functional defects in monocytes play a key role in the pathophysiology of sepsis-induced immune-suppression owing to a decreased release of pro-inflammatory cytokines, low human leukocyte antigen-DR isotype (HLA-DR) surface expression and reduced antigen presentation capacity [4]. Several clinical studies show an association of the low levels of HLA-DR expression on circulating monocytes with morbidity and mortality of septic patients and increased susceptibility to contracting secondary/nosocomial infections [5-7].

Similar to the phenomenon observed in monocytes, changes in immune function also involved lymphocytes [8]. Sepsis causes massive apoptosis of cluster of differentiation (CD) $4^{+}$and $\mathrm{CD} 8^{+} \mathrm{T}$ cells as well as B cell [9-11]. Lymphocyte apoptosis is a key factor in the development of immune paraly- 
sis because it also impairs the activity of innate immunity cells, which are the most potent antigen-presenting cells (APCs), contributing to the progression of immunological imbalance of septic patients [12]. Surviving $\mathrm{CD}^{+}$and $\mathrm{CD}^{+} \mathrm{T}$ cells develop an "exhausted" phenotype because of the prolonged exposure to excessive pro- and anti-inflammatory cytokines. Several studies show that Th1- and Th2-associated cytokine production is reduced during and long after sepsis resolution $[4,13]$; in particular Boomer et al [14] showed a profound suppression of interferon (IFN)- $\gamma$ and TNF- $\alpha$ production. T cells exhaustion also includes increased expression of programmed cell death-1 (PD1) on $\mathrm{CD}^{+} \mathrm{T}$ cells and of programmed cell death ligand 1 (PDL1) on macrophages and a marked reduction in T-bet and GATA3 expression, two transcription factors that modulate Th1 and Th2 cell response, respectively [15]. Instead, in the immune paralysis state, an increase of regulatory $\mathrm{T}$ cells $(\mathrm{T}$ reg) has been observed [16]. $\mathrm{T}$ reg cells are more resistant to sepsis-induced apoptosis than other $\mathrm{T}$ cell subpopulations; their higher number in sepsis patients impairs immunity and contributes to secondary infections and mortality by acting both on innate and adaptive immune cells [9], increasing immune-suppressive state.

Evaluation of immunophenotyping of whole peripheral blood represents a good marker to establish immune cells anergy, determine immune paralysis of innate and adaptative immunity and possibly monitor the effectiveness of immunemodulatory therapies $[16,17]$.

In the present study, we conducted a flow cytometrical analysis of the functional changes in monocytes HLA-DR molecule, as marker of their activation or paralysis in septic patients, together with the evaluation of the T cell-driven adaptative immunity by residual capacity of IFN- $\gamma$ production during sepsis, in correlation with clinical outcome of disease.

\section{Materials and Methods}

\section{Patients}

The present study has been conducted on 55 patients hospitalized in different medical and surgical wards of the Vito Fazzi Hospital of Lecce (Italy) and 20 controls (not hospitalized healthy individuals). Patients were enrolled according to inclusion criteria: adult age (equal or more than 18); clinical suspicions of bacterial infection (patients were enrolled with high procalcitonin (PCT) plasmatic levels (PCT $>0.5 \mathrm{ng} / \mathrm{mL}$ ); sepsis diagnosis was established according to the Sepsis-3 definition of the Society of Critical Care Medicine and the European Society of Intensive Care Medicine [18]; the presence of infection was confirmed by microbiological assays. Exclusion criteria were the presence of human immunodeficiency virus (HIV) infection, infectious hepatitis and other acute or chronic viral infections.

Patients with confirmed sepsis $(\mathrm{n}=39$, positive blood culture $(\mathrm{BC}+))$ have been monitored for 1 week after admission. Long hospitalized patients (16 out of 39 ) were monitored for 4 weeks $(\mathrm{sBC}+)$.

For each patient, clinical and biological variables were collected. These included demographic characteristics (age and gender), microbiological findings (infection source and the identified microorganisms) and routine markers of inflammation as C-reactive protein (CRP), complete blood cell count and biochemical markers; the Sequential Organ Failure Assessment (SOFA) score has been calculated for septic patients. SOFA score was determined by using biochemical and clinical parameters of each patient. SOFA score is based on the degree of dysfunction of six organ systems: respiratory failure is assessed by fraction of inspired oxygen and partial pressure of oxygen $\left(\mathrm{PaO}_{2}\right)$, coagulation score by platelets count, liver score by total bilirubin, neurological score by Glasgow Coma Scale, cardiovascular function by mean of arterial pressure and use of vasopressors, renal impairment by serum creatinine or urine output. To each organ system, a point value from 0 (normal) to 4 (high degree of dysfunction/failure) is assigned. The SOFA score ranges from 0 to 24; higher value indicates greater organ failure and worst prognosis.

The Institutional Review Board (IRB) approval was not applicable. This study was conducted in compliance with the ethical standards of the responsible institution on human subjects as well as with the Helsinki Declaration. Written informed consent was obtained from the patients, or if not possible, from their relative as designed by ethic committee.

\section{Hematological and biochemical investigation}

Complete blood count was performed on Sysmex XN-3100 automatic hematology analyzer (Sysmex, Kobe, Japan) based on the fluorescence flow cytometry and hydrodynamically focused impedance. Cell population data (CPD) for the leukocyte sub-populations (neutrophils (NE); monocytes (MO); lymphocytes (Ly)) were obtained using flow cytometry in the WDF channel of the XN-3100 from the optical signals related to internal complexity along the $\mathrm{X}$ axis (side scatter, $\mathrm{SSc}$ ), fluorescence related to nucleic acid content along the $\mathrm{Y}$ axis (SFl), forward scatter (FSc) proportional to cell size in the Z axis. Parameters related to dispersion of values around the mean are expressed according to distribution width; as range of the distribution of the major population, as detailed in the study of Urrechaga et al [19].

Plasmatic levels of PCT were determined by enzymelinked fluorescence assay (ELFA) with VIDAS ${ }^{\circledR}$ B.R.A.H.M.S. PCT $^{\mathrm{TM}}$ system (Biomerieux, Marcy-l'Etoile, France) according to manufacturer recommendations and expressed in $\mathrm{ng} / \mathrm{mL}$.

Serum levels of biochemical parameters analyzed were performed on the Cobas ${ }^{\circledR} 8000$ analyzer according to manufacturer's directions (Roche Diagnostic GmbH, Germany).

\section{Microbiological cultural assay}

Microbial cultures were used to identify the organism present in biological specimens derived from different sites (respiratory tract, urinary tract, abdomen, and others) according to clinical suspicion by standardized microbiological cultural assays procedures. 
Blood cultures were performed in patients with clinical symptoms of bloodstream infections prior the administration of antimicrobial therapy. For each patient, two bottles sets were used for each septic episode; approximately $10 \mathrm{~mL}$ of blood was inoculated in the aerobic and anaerobic bottle (BACT/ ALERT Culture Media, Biomerieux, Marcy-l'Etoile, France); the bottles were entered in the BACT/ALERT 3D system for the incubation and measure of the color change in response to shift in $\mathrm{pH}$ as a result of rising of carbon dioxide $\left(\mathrm{CO}_{2}\right)$ levels produced by microorganisms. In positive samples, bacteria and yeasts were identified on the Vitek 2 system (Biomerieux, Marcy-l'Etoile, France), according to manufacturer directions.

\section{Flow cytometrical quantification of HLA-DR in mono- cytes}

One hundred $\mu \mathrm{L}$ of ethylenediaminetetraacetic acid (EDTA) anticoagulated whole blood from patients (after 3 - 4 days from sepsis diagnosis) or controls were incubated with the following combination of monoclonal antibodies: $20 \mu \mathrm{L}$ of anti CD14-PE (BD, clone MфP9) and $20 \mu \mathrm{L}$ of anti HLA-DRAPC-Cy7 (BD, clone L243) for $30 \mathrm{~min}$ in the dark. All samples were stained within $1.5 \mathrm{~h}$ after blood collection.

After staining, red blood cells were lysed for 15 - $20 \mathrm{~min}$ with 1:10 dilution of BD FACS ${ }^{\text {TM }}$ lysing solution (containing $30 \%$ diethylene glycol, $9.99 \%$ formaldehyde and $3.51 \%$ methanol), and centrifugated for $5 \mathrm{~min}$ at $300 \times \mathrm{g}$. Cell pellet was resuspended in $450 \mu \mathrm{L}$ of BD FACSFlow ${ }^{\mathrm{TM}}$ sheath fluid solution and acquired on a BD FACS CANTO II flow cytometer.

A minimum of 3,500 monocyte events were recorded for each sample based on a gate created on scatter plot of CD14PE vs. side scattered light signals (SSC). HLA-DR expression on blood monocytes (mHLA-DR) was reported as a percentage and mode of fluorescence intensity (MFI) of HLA-DR positive monocytes out of the total $\mathrm{CD}_{1} 4^{+}$monocyte population. In setting conditions, we used also monoclonal antibodies anti CD45 and markers for further leucocytes population than monocytes (as anti CD3 for lymphocytes T, anti CD19 for lymphocytes B or other specific markers of granulocytes lineage in combination with dimensional parameters), and we confirmed the accurate gating of monocytes even in absence of additional markers (data not shown).

Absolute quantification of mHLA-DR per cell was performed by anti-HLA-DR/anti-monocyte reagent (BD, Quantibrite Anti-HLA-DR/Anti-Monocyte kit), a combination of anti-HLA-DR PE ( $\geq 95 \%$ 1:1 PE:mAb ratio, cloneL243) and anti-monocyte PerCP-Cy5.5 (CD14, clone M $\varphi$ P9, and CD64, uniquely recognized by cyanine dyes). Fluorescence quantitation was performed after calibration by fluorescent beads with known amount of HLA-DR per bead (BD, Quantibrite PE Fluorescence Quantitation Kit).

Data were analyzed by BD Facs DIVA 8.0.1 software (BD).

\section{IFN- $\gamma$ release assay}

The IFN- $\gamma$ release assay (IGRA) was performed by stimulating blood cells with specific microbial antigens and quantifying IFN- $\gamma$ by QuantiFERON ${ }^{\circledR}$-TB Gold Plus Kit on LIAISON ${ }^{\circledR}$ XL (DiaSorin, Saluggia Italy). Briefly, $1 \mathrm{~mL}$ lithium-heparin anticoagulated whole blood from 16 septic patients and 20 controls was incubated $48 \mathrm{~h}$ at $37{ }^{\circ} \mathrm{C}$ in presence of phytohemagglutinin (PHA, positive control) or $100 \mu \mathrm{L}$ of bacterial cell lysate from Staphylococcus aureus ( $S$. aureus) culture, (corresponding to approximately $10-15 \times 10^{6}$ microbial cells per tube) or $100 \mu \mathrm{L}$ of cell lysate from Candida albicans $(C$. albicans) culture, (corresponding to approximately $10-15 \times$ $10^{6}$ microbial cells per tube) or no stimulus (negative control). After incubation, the samples were centrifuged for $15 \mathrm{~min}$ at $3,000 \times \mathrm{g}$ to separate cells from plasma. Concentrations of IFN- $\gamma$ released by stimulated T-cell were determined by chemiluminescent immune assay (CLIA) by LIAISON ${ }^{\circledR}$ XL analyzer (DiaSorin) according to manufacturer's recommendation, and expressed in $\mathrm{UI} / \mathrm{mL}$ on the basis of a calibration curve obtained with known amount of IFN- $\gamma$. Values of IFN- $\gamma$ $>0.35 \mathrm{UI} / \mathrm{mL}$ in presence of mitogens indicate $\mathrm{T}$ cells activation; for positive control (PHA) the cut-off was $>0.5 \mathrm{UI} / \mathrm{mL}$.

\section{Statistical analysis}

Statistical analysis was performed by MedCalc v19.9.1 statistical software and data were showed as the mean \pm standard error of the mean (SEM).

The Student's $t$-test was used for comparison between patients and controls; statistically significant differences were established according to $\mathrm{P}$ value $(<0.05)$.

\section{Results}

In the present study we analyzed a population of 55 hospitalized patients with high plasmatic level of PCT $>0.5 \mathrm{ng} / \mathrm{mL}$ (hPCT patients) and early suspicion of sepsis, and 20 not hospitalized healthy individuals, as controls (Table 1).

The 55 hPCT patients had positive cultural assay: 39 of them had clinical evidences of sepsis and $\mathrm{BC}+$, responding to Sepsis-3 International Consensus Definition [18]; remaining 16 patients, denoted as BC-, had positive cultural assay in different sites (respiratory tract, genitourinary tract, abdominal cavity, and skin). Gram-negative bacteria were identified in $74 \%$ of positive blood cultures.

Among $\mathrm{BC}+$, a subgroup of 16 patients $(\mathrm{sBC}+)$ was monitored for 4 weeks (T0 - T5); five of them died between the second and third week. The SOFA score of alive patients was 4 \pm 0.91 while it was $11 \pm 2.60$ in deceased patients.

Biochemical and hematological parameters were monitored for all $\mathrm{BC}+$ patients at admission (time 0, T0) and after 1 week from diagnosis (time 2, T2). The whole group of septic patients $(\mathrm{BC}+)$ was retrospectively categorized into two groups characterized by better clinical outcome (group BO, $\mathrm{n}$ $=27$ ) or worst clinical outcome (group WO, $\mathrm{n}=12$ ) based on time of hospitalization, recovery time from primary infection, appearance of secondary/nosocomial infections and death (Fig. 1). The whole group was monitored for 1 week (T0 - T2). 
Table 1. Characteristics of Analyzed Patients

\begin{tabular}{lllll}
\hline & $\begin{array}{l}\text { Healthy controls } \\
(\mathbf{C t r l}, \mathbf{n = 2 0})\end{array}$ & $\begin{array}{l}\text { Presence of infection } \\
(\mathbf{B C}-\mathbf{n}=\mathbf{1 6})\end{array}$ & $\begin{array}{l}\text { Confirmed sepsis } \\
(\mathbf{B C}+, \mathbf{n}=\mathbf{3 9}(\mathbf{B O}=\mathbf{2 7} ; \mathbf{W O}=\mathbf{1 2}))\end{array}$ & $\begin{array}{l}\mathbf{4} \text { weeks follow-up } \\
(\mathbf{s B C}+, \mathbf{n}=\mathbf{1 6}(\mathbf{d e c e a s e d :} \mathbf{5} / \mathbf{1 6}))\end{array}$ \\
\hline Age (years) & $53 \pm 4$ & $68 \pm 3$ & $67 \pm 3$ & $72 \pm 3$ \\
Gender (male/female) & $6 / 12$ & $11 / 5$ & $18 / 21$ & $6 / 10$ \\
Blood culture & - & - & + & + \\
Other specimens culture $^{\mathrm{a}}$ & - & + & - & - \\
\hline
\end{tabular}

aOther specimens culture: samples from respiratory and urogenital sites, serous cavities, and skin. BC+: patients with positive blood culture; BC-: patients with positive cultural assay in different sites; BO: septic patients with better clinical outcome; WO: septic patients with worst clinical outcome; $\mathrm{SBC}+$ : long hospitalized septic patients monitored for 4 weeks. Data are presented as mean \pm SEM or $n$. SEM: standard error of the mean.

\section{Analysis and quantification of HLA-DR monocytes sur- face expression in control and septic patients}

mHLA-DR expression was analyzed 3 - 4 days after diagnosis of sepsis in 39 septic patients $(\mathrm{BC}+)$ by flow cytometrical analysis (Fig. 2). Percentage value of mHLA-DR in 16 non-septic patients (BC-) showed no significant difference compared to controls (99.1 $\pm 0.3 \%$ vs. $99.4 \pm 0.2 \%$, with $\mathrm{P}=0.3)$. $\mathrm{BC}+$ septic patients showed significantly lower percentage of mHLA-DR $(94.3 \pm 2.29 \%)$ compared to both healthy controls (with $\mathrm{P}<$ 0.05 ) and to $\mathrm{BC}$ - patients (with $\mathrm{P}<0.05$, Fig. 2). No statistical significance was observed for mHLA-DR MFI between $\mathrm{BC}+$, $\mathrm{BC}$ - and control patients.

The worst conditions subgroup of patients, WO, was characterized by higher incidence of secondary/nosocomial infections, often polymicrobial, long time hospitalization $(>30$ days) and statistically significant lower percentage of mHLA-
DR $(88.4 \pm 4.5 \%)$ compared with better outcome patients, BO $(98.6 \pm 0.3 \%, \mathrm{P}=0.05)$. WO patients showed statistically significant lower MFI of mHLA-DR (1,193.6 \pm 219.6 vs. 2,819.4 \pm 591.1 of $\mathrm{BO}$ patients, $\mathrm{P}=0.022$ ). Florescence intensities of WO vs. BO were significantly different also when evaluated as median $(1,623.7 \pm 416.3$ vs. $2,730.8 \pm 332.5)$ or mean values $(2,344.0 \pm 590.2$ vs. $3,837.4 \pm 398.1)$, with $\mathrm{P}=0.05$.

Interestingly, WO patients did not show a tendency towards restoration of normal values of white blood cells, platelets, monocytes, neutrophils and lymphocytes counts like BO patients (Fig. 1) or BC- patients, which however exhibited more stable hematological parameters than $\mathrm{BC}+$ (data not shown).

We further monitored $16 \mathrm{BC}+$ patients hospitalized for 4 consecutive weeks $(\mathrm{sBC}+)$ in order to quantify absolute number of HLA-DR molecules per monocyte and evaluate the activation/anergy state of monocytes in relation to clinical outcome and survival/mortality of patients. Five out of 16 patients
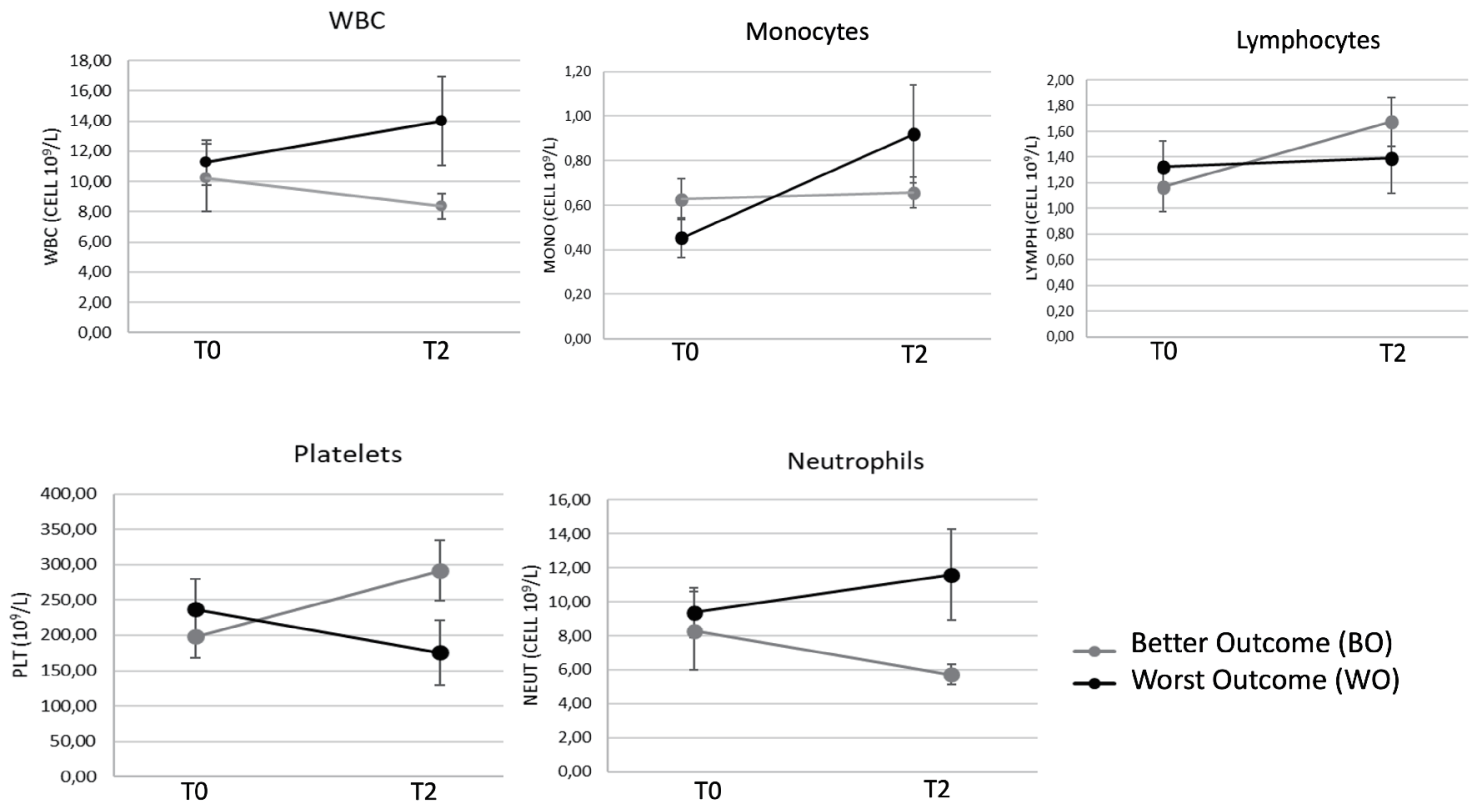

Figure 1. Hematological parameters of 39 patients with confirmed sepsis $(\mathrm{BC}+)$ after 1-week hospitalization. White blood cells (WBC), monocytes, lymphocytes, neutrophils and platelets (PLT) count were monitored at admission (time 0 , T0) and after 1 week from diagnosis (time 2, T2). Patients were sub-grouped in better outcome (BO) and worst outcome (WO) according to clinical severity. Data are presented as mean \pm SEM. Statistical analysis: unpaired Student's $t$-test of WO vs. BO values. SEM: standard error of the mean. 


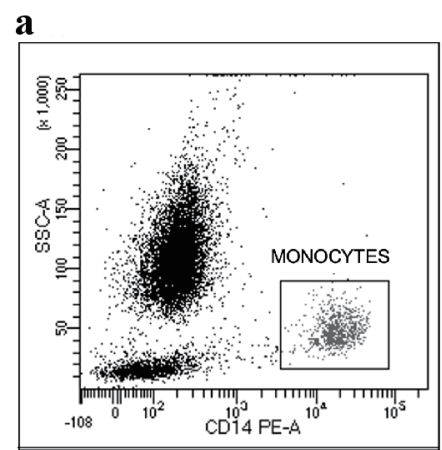

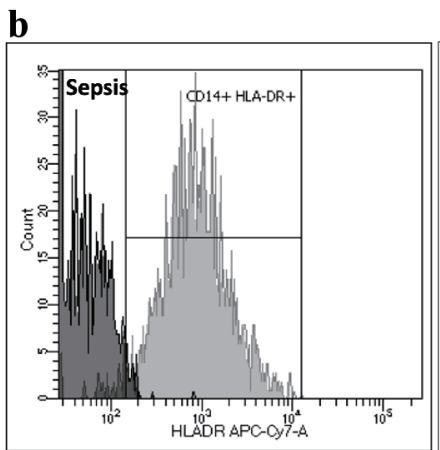

Ctrl BC-

c

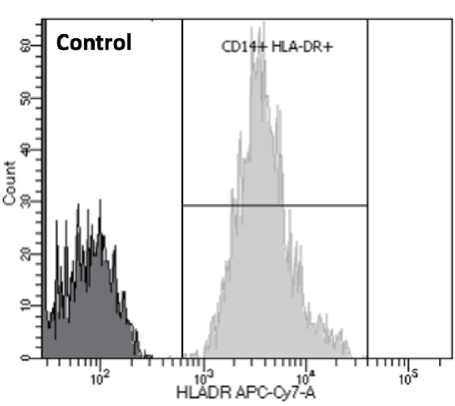

$\mathrm{BC}+$

\begin{tabular}{|c|c|c|c|c|}
\hline \multirow[t]{3}{*}{$\% \mathrm{CD} 14^{+} \mathrm{HLA}-\mathrm{DR}^{+}$} & \multirow[t]{3}{*}{$99.4 \% \pm 0.2$} & \multirow[t]{3}{*}{$99.1 \% \pm 0.3$} & \multicolumn{2}{|c|}{$94.3 \%{ }^{\S \#} \pm 2.29$} \\
\hline & & & $\begin{array}{l}\text { Better Outcome } \\
\text { (BO) }\end{array}$ & $\begin{array}{l}\text { Worst Outcome } \\
\text { (WO) }\end{array}$ \\
\hline & & & $98.6 \%^{\#} \pm 0.3$ & $88.4 \% * \# \pm 4.5$ \\
\hline $\begin{array}{l}\text { MODE OF FLUORESCENCE } \\
\text { INTENSITIES (MFI) }\end{array}$ & $\begin{array}{l}2206.5 \pm \\
118.0\end{array}$ & $\begin{array}{c}2143.4 \pm \\
39.2\end{array}$ & $2819.4 \pm 591.1$ & $1193.6 * \# \pm 219.6$ \\
\hline
\end{tabular}

${ }^{s}: p<0.05 B C+v s B C-,{ }^{*}: p<0.05 B C+v s C t r l, *: p<0.05$ WO vs BO.

$B C+$, patients with positive blood culture; $B C-$, patients with other positive cultural assays.

Figure 2. HLA-DR monocytes surface expression in $B C+, B C$ - and control (Ctrl) patients (total $n=55$ ). (a) Flow cytometrical analysis of monocytes gated on scatter plot of CD14-PE vs. side scattered light signals (SSC). (b, c) Histogram plot of HLA-DRAPC-Cy7 among CD14+ gated monocytes. Values of HLA-DR expression percentage and mode of fluorescence intensity are indicated below graphs for different groups of patients and controls. Data are presented as mean \pm SEM. Statistical analysis: unpaired Student's $t$-test of BC+ vs. BC- values ( $\$ P<0.05)$; $B C+$ vs. Ctrl values ( $\left.{ }^{\#} P<0.05\right)$; WO vs. BO values ( $\left.{ }^{*} P<0.05\right)$. HLADR: human leukocyte antigen-DR isotype; $\mathrm{BC}+$ : patients with positive blood culture; BC-: patients with other positive cultural assays; WO: patients with worst clinical outcome; BO: patients with better clinical outcome.

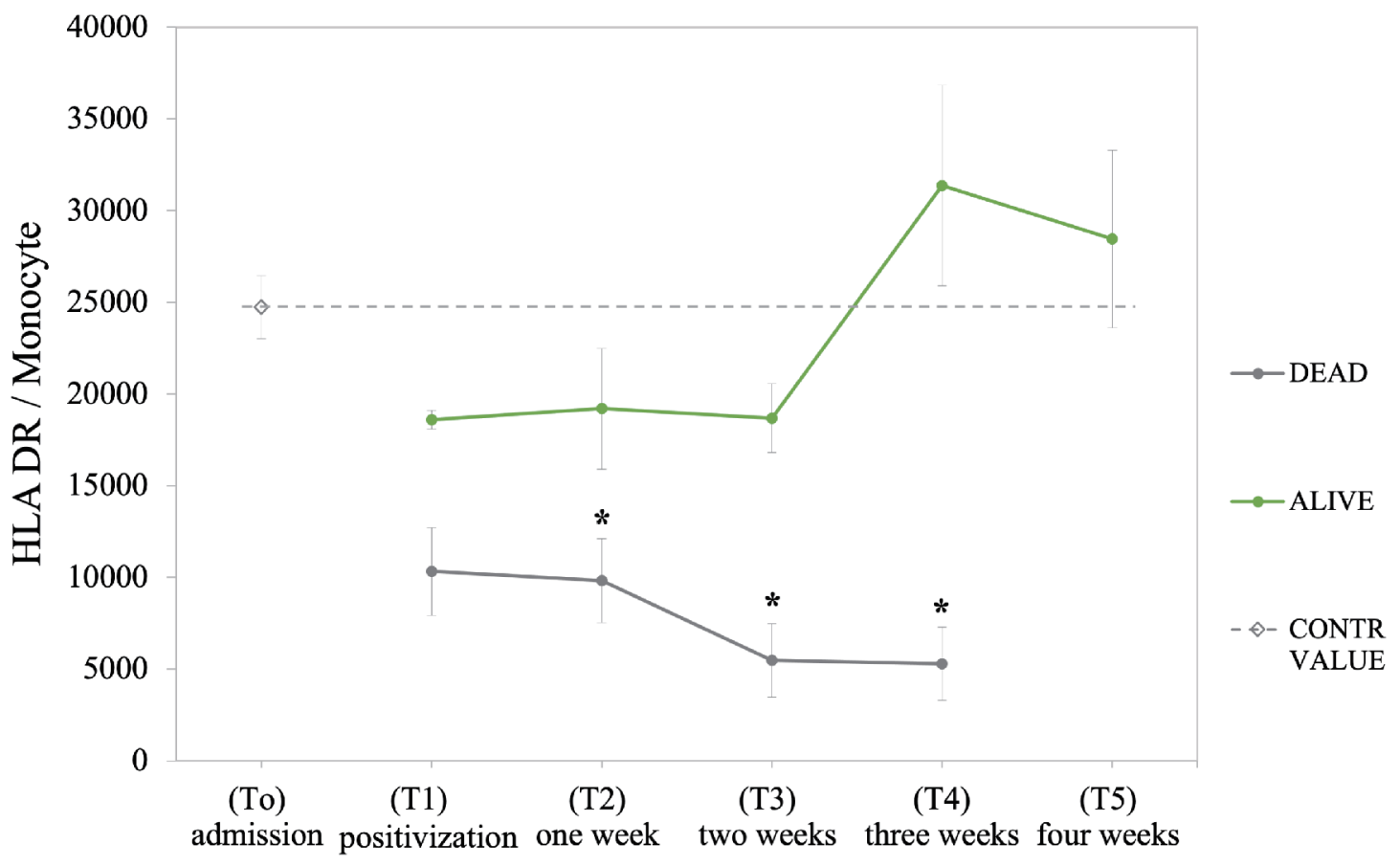

Figure 3. Quantification of HLA-DR per monocyte in the sub-group of 16 hospitalized septic patients (sBC+) monitored for 4 weeks following septic diagnosis. Patients were classified according to clinical outcome (alive and dead patients); the dashed line indicates value obtained for healthy individuals. Data are presented as mean \pm SEM. Statistical analysis: unpaired Student's $t$-test of dead vs. alive values ( $\left.{ }^{*} \mathrm{P} \leq 0.05\right)$. HLA-DR: human leukocyte antigen-DR isotype; SEM: standard error of the mean. 
died between the second and third week. We found that the absolute count of mHLA-DR was significantly lower in patients with adverse outcome compared to alive (9,824 vs. 19,207 HLA-DR molecules/monocyte, $\mathrm{P}=0.05$, after the first week from sepsis diagnosis; 5,956 vs. 18,862 HLA-DR molecule/ monocyte, $\mathrm{P}<0.05$, after the second week; 5,583 vs. 31,367 HLA-DR molecule/monocyte, after the third week, $\mathrm{P}<0.05$ ). A clear decreasing trend of HLA-DR molecules per monocyte (Fig. 3) was observed during time in deceased patients with respect to survivals (mortality has been evaluated at the 28th day, all deceased patients died before the fourth week).

\section{Analysis of CPD}

Analysis of CPD for monocytes revealed increased value for $\mathrm{X}, \mathrm{Y}, \mathrm{Z}$ parameters (intracellular complexity, $\mathrm{X}$; nucleus florescence, Y; cellular dimensions, Z) for $\mathrm{sBC}+$ septic individuals compared to controls, at the first week of follow-up. The increase was more pronounced in not survived individuals with $\mathrm{P} \leq 0.05$ compared to alive patients (Table 2 ). The previously reported [19] increase of monocyte dispersion width (MDW) observed in sepsis (for the width of each parameter, WX, WY, WZ) was found only in survived septic patients. However, in deceased patient, the nucleic acid content (Y) appeared reduced at the second week and corresponded to an increased MDW (MO-WY). Interestingly, this finding appears in accordance to the observed decline in mHLA-DR expression in deceased septic patient from the second week of follow-up.

Lymphocytes (Ly) showed similar CPD trend with increased intracellular complexity (Ly-X) during sepsis compared to control individuals, while nuclear florescence (LyY) was decreased compared to control; we found significant decrease of nuclear fluorescence in deceased patients vs. survived $(\mathrm{P}=0.05)$ at the third week of sepsis follow-up (Table 2$)$. Increased lymphocyte dispersion width (LDW) was observed during sepsis, for deceased individuals the increase of Ly-WX and Ly-WY was observed with a delay of $1-2$ weeks. This is in accordance with the almost inexistent $\mathrm{T}$ lymphocytes activation and IFN- $\gamma$ production in presence of mitogens in deceased septic patients compared to survived individuals (Table 2).

\section{T cells response to mitogens and IFN- $\gamma$ production}

T cells activation was investigated in $\mathrm{sBC}+$ patients, during 4 consecutive weeks, through the evaluation of IFN- $\gamma$ released after lymphocytes stimulation with PHA, S. aureus and C. albicans. In presence of PHA, we observed that IFN- $\gamma$ levels were significantly lower in patients with unfavorable outcome compared to survived patients $(1.43 \pm 0.27 \mathrm{UI} / \mathrm{mL}$ vs. $7.66 \pm$ $0.96 \mathrm{UI} / \mathrm{mL}$, respectively, with $\mathrm{P}<0.001$, after 1 week from sepsis diagnosis; $1.15 \pm 0.64 \mathrm{UI} / \mathrm{mL}$ vs. $8.82 \pm 0.54 \mathrm{UI} / \mathrm{mL}$ with $\mathrm{P}<0.05$, after second week; $0.54 \pm 0.32 \mathrm{UI} / \mathrm{mL}$ vs. 6.62 $\pm 0.93 \mathrm{UI} / \mathrm{mL}$ with $\mathrm{P}<0.05$, after third week, Fig. 4). S.aureus and $C$. albicans did not stimulate $\mathrm{T}$ cells response in patients with lethal clinical outcome. IFN- $\gamma$ levels were very low $(0.03$ $\pm 0.009 \mathrm{UI} / \mathrm{mL}$ and $0.07 \pm 0.03 \mathrm{UI} / \mathrm{mL}$, respectively, after the first week from sepsis diagnosis; $0.03 \pm 0.01 \mathrm{UI} / \mathrm{mL}$ and 0.05 $\pm 0.01 \mathrm{UI} / \mathrm{mL}$, after second week; $0.04 \pm 0.03 \mathrm{UI} / \mathrm{mL}$ and 0.04 $\pm 0.01 \mathrm{UI} / \mathrm{mL}$, after third week) compared to survivors $(2.08 \pm$ $1.10 \mathrm{UI} / \mathrm{mL}$ and $1.22 \pm 0.88 \mathrm{UI} / \mathrm{mL}$, respectively, after the first week; $1.67 \pm 1.10 \mathrm{UI} / \mathrm{mL}$ and $0.51 \pm 0.35 \mathrm{UI} / \mathrm{mL}$, after second week; $1.71 \pm 1.05 \mathrm{UI} / \mathrm{mL}$ and $0.12 \pm 0.02 \mathrm{UI} / \mathrm{mL}$, after third week). Although the strong decline of $\mathrm{T}$ cells response to mitogens observed in deceased patients, we did not find a statistically significant difference between dead and alive because of the small dimension of the analyzed sample (Fig. 4).

\section{Discussion}

Delayed restoration of immunologic homeostasis between pro- and anti-inflammatory responses determines an impaired immune reaction following sepsis and higher risk for adverse outcome including secondary infection and death [4]. The innate immune cells (including monocytes) are involved in the initial immunologic response to critical illness; the adaptative immune response (lymphocytes-driven) is predominant in the subacute phase of illness. In the present study, we analyzed activation state of monocytes and lymphocytes in the immunosuppressive phase of sepsis in critically ill patients.

As known, monocytes normally recognize and process the pathogen and present the antigens on their cell surface via HLA-DR to T helper cells; once activated, monocytes secrete pro-inflammatory cytokines amplifying the immune response, while T helper cells interact and activate B cells to proliferate, differentiate and secrete specific antibodies. When critical condition advances, a parallel anti-inflammatory process leading to the immune-suppressed state determines a reduced monocytes antigen presenting capacity and releasing of pro-inflammatory cytokines in response to bacterial compounds [6, 20, 21].

In this context, we examined mHLA-DR expression during sepsis, and we found a significant reduction of its percentage value in septic patients $(\mathrm{BC}+)$ compared to controls and to BC-patients (Fig. 2). Notably, the subgroup of septic patients with worst outcome (WO) exhibited a significant reduction of mHLA-DR percentage and MFI (Fig. 2), in agreement with the worsening of hematochemical markers at the first week; opposite to the restoration of same parameters in BO patients (Fig. 1). After the first week from sepsis diagnosis, in patients with fatal prognosis the absolute number of HLA-DR molecules per monocytes was reduced to around $50 \%$ of the value found in patients with positive outcome; at the third week, it decreased to less the $20 \%$ (Fig. 3). This finding correlates with the variation of monocytes CPD in deceased patients compared to those survived to sepsis. In fact, we found decreased nucleic acid content from the second week of sepsis and increased MDW in deceased patients (Table 2).

As we have previously reported, during the first phase of sepsis there is a progressive polarization of monocytes to a pro-inflammatory phenotype characterized by the expansion of the $\mathrm{CD} 14^{++} \mathrm{CD} 16^{+}$subpopulation (intermediate monocytes, mainly a pro-inflammatory subset) and the decrease of classical CD14 ${ }^{++}$CD16- monocytes subpopulation [22]. CD16 ${ }^{+}$monocytes are known to be involved in antigen processing and presen- 


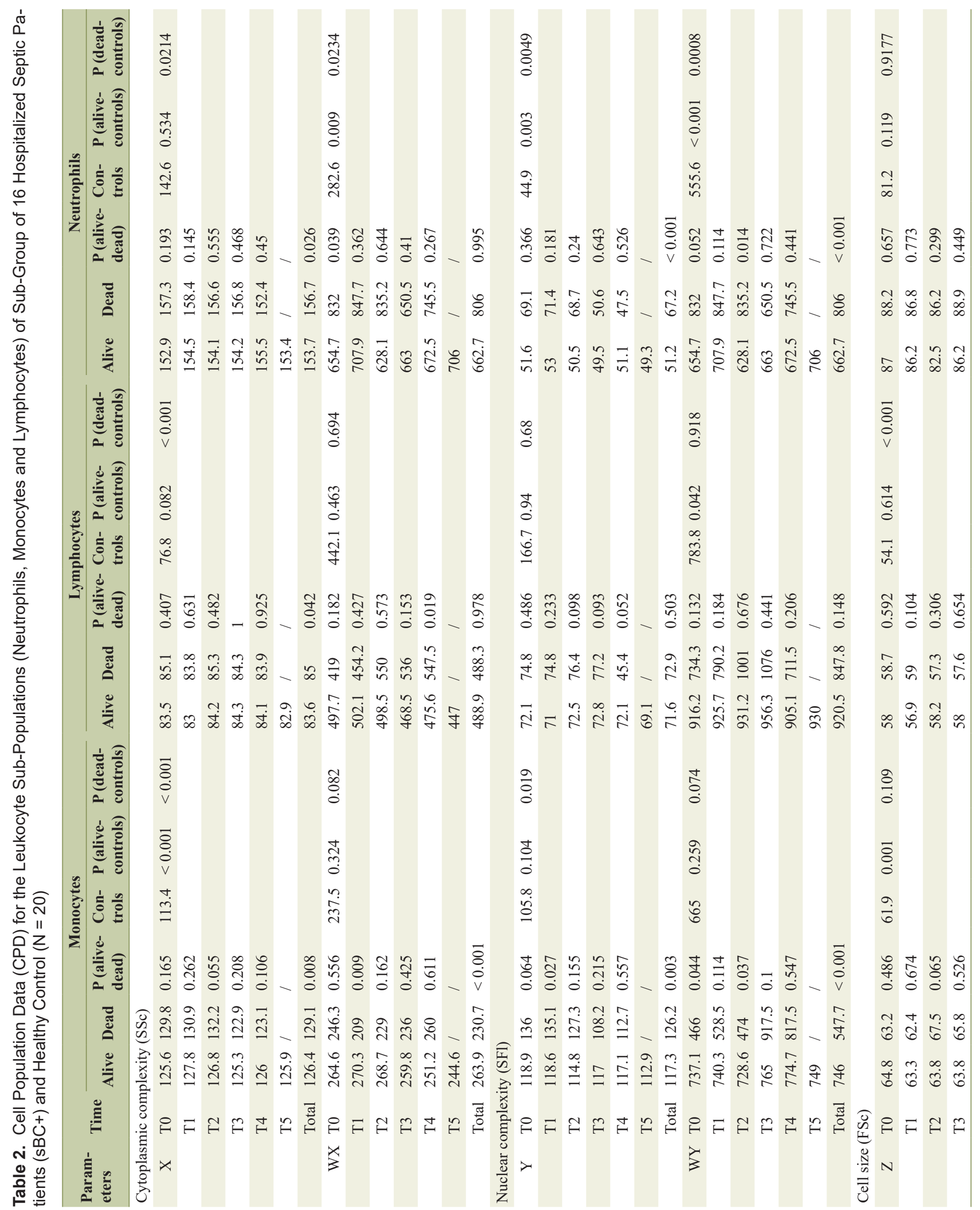




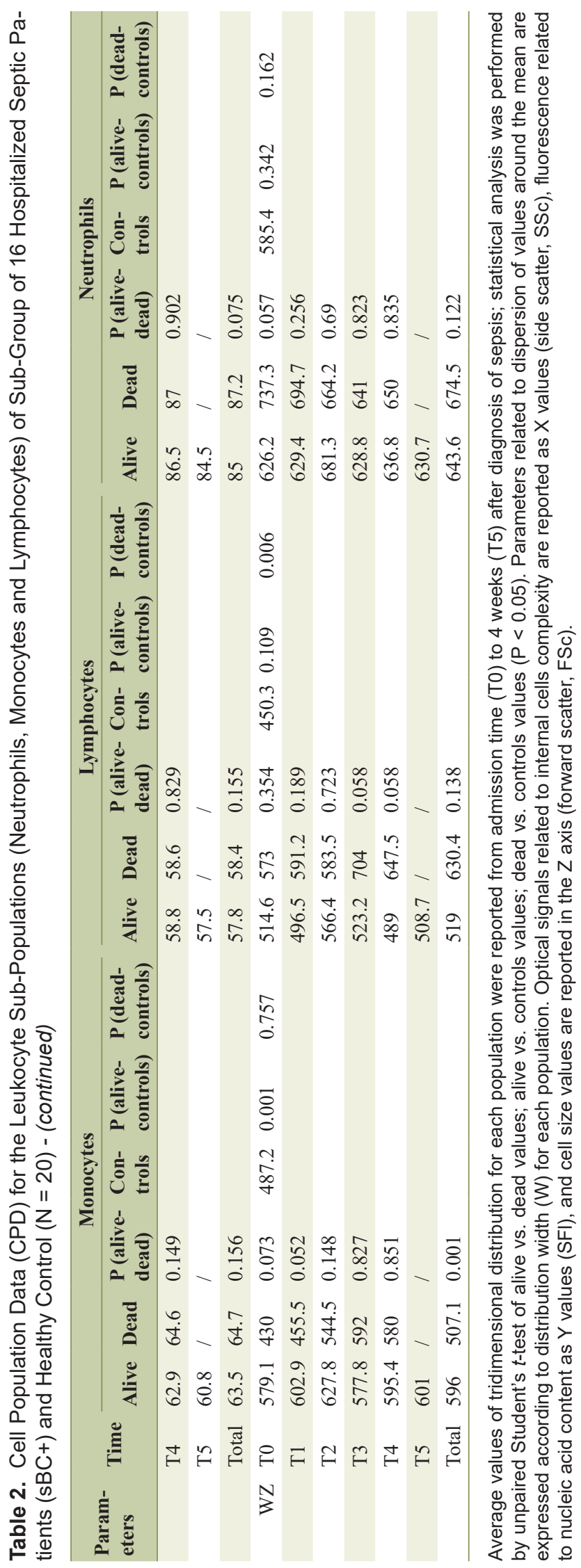

tation and trans-endothelial migration [23], and to be increased during inflammation (e.g., cancer, sepsis and stroke), infections such as HIV, tuberculosis and other pathogens [24-31]. Other studies demonstrated intermediate monocytes involvement in inflammatory response by evidencing intermediate monocytes triggered expression of molecules, which promote their migration into the lesions, where increased levels of their ligand are present [32], also facilitated by increased permeability and endothelial activation [33]. Nevertheless, after the first intense inflammatory response, subsequent immunocompromised state is characterized by an activated anti-inflammatory response in order to restoring immune homeostasis $[4,34]$. Present results confirmed that during sepsis, monocytes undergo functional and metabolic reprogramming by reducing the levels of HLA-DR surface expression (Figs. 2, 3) and internal complexity (Table 2), the immunosuppressed phenotype observed for patients with fatal prognosis contributes to the poorest clinical outcome.

From our results, impaired monocytes activation during the innate immune response and reduced mHLA-DR appear to be good markers for classification of adverse clinical outcome in septic patients. Further impairment of adaptive immune response with lymphocyte apoptosis and lymphopenia is associated with increased mortality and secondary infection risk [35-37]. We observed a substantial drop of lymphocytes absolute count in WO patients after 1 week (Fig. 1), while both BO patients and BC- patients showed a more stable trend and better outcome. In the subgroup of five deceased patients, lymphocytes were considerably lower than survived patients at the stage of the diagnosis of sepsis and during following 4 weeks (even lower than the value found in whole group of WO BC+ patients). The absolute count of lymphocytes was $0.48 \pm 0.26 \times 10^{9} \mathrm{cell} / \mathrm{L}$ at blood culture positivization, $0.69 \pm$ $0.29 \times 10^{9} \mathrm{cell} / \mathrm{L}$ after 1 week, $0.74 \pm 0.01 \times 10^{9} \mathrm{cell} / \mathrm{L}$ after 2 weeks (with P, respectively, 0.03, 0.04, and 0,06). Moreover, lymphocytes showed different CPD behavior (variation width for parameters of intracellular complexity and nuclear florescence) in deceased patients compared with patients with good prognosis. Again, for deceased individuals these parameters were correlated with a state of anergy, reduced activation and failure of IFN- $\gamma$ production in presence of microbial mitogens, as $S$. aureus and C. albicans, or PHA at the diagnosis of sepsis and during following weeks (Fig. 4).

Other hematological parameters showed an expected increase of absolute numbers of neutrophils and decrease of platelets in deceased patients during the 4-week observation (data not shown). CPD data for neutrophils showed a consistent trend of increasing values (internal complexity, nuclear fluorescence and their related variation width) for dead septic patients compared to alive, especially at the first week; differences were significative compared to control individuals.

Our data demonstrated that both lymphopenia and lymphocytes impaired functional activity were associated with increased immune dysfunction and higher sepsis severity. Currently, there are several reports showing the altered lymphocytes immune function in sepsis. Feuerecker et al [38] observed reduced IFN- $\gamma$ and other pro-inflammatory cytokines levels after in vitro stimulation of lymphocytes from patients affected by severe sepsis or septic shock, as well as ex vivo stimulation with IL-10 [39]. Our results showed that sepsis-in- 
PHA



S. aureus



C. albicans



Figure 4. Interferon-y released by lymphocytes (IFN-y release assay (IGRA) test) in presence of mitogens: (a) Positive control (PHA). (b, c) Microbial cells lysate (S. aureus and C. albicans) in the group of 16 hospitalized septic patients monitored for the first 3 weeks following septic diagnosis. Patients were classified according to clinical outcome (alive and dead patients). The fourth week was not reported in the graph because all deceased patients died before the 28th days. Data are presented as mean \pm SEM. Statistical analysis: unpaired Student's $t$-test of dead vs. alive values $\left({ }^{\star} P \leq 0.05 ;{ }^{* \star \star} P<0.001\right)$. SEM: standard error of the mean; IFN: interferon; PHA: phytohemagglutinin; S. aureus: Staphylococcus aureus; C. albicans: Candida albicans.

duced defects in innate immune cells contribute to immune paralysis along with lower ability of adaptive immune responses. The phenotypic shift toward down regulation of multiple immune response pathways in both monocytes and $\mathrm{T}$ cell [40] suggested more susceptibility to secondary infections, and consequently, late-phase mortality.

In conclusion the present study evidenced the impairment of the antigen presentation process and $\mathrm{T}$ cell IFN- $\gamma$ release and its worsening during the 4-week observation of hospitalized patients with consequent immune paralysis due to sepsis and severe outcome. The analysis of cells anergy and immune paralysis is an important tool in order to better understand the pathophysiological mechanisms of sepsis, the correlation with clinical outcome and development of new immune-monitoring strategies and effective immunotherapies.

\section{Acknowledgments}

None to declare.

\section{Financial Disclosure}

None to declare.

\section{Conflict of Interest}

The authors declare that they have no conflict of interest.

\section{Informed Consent}

Informed consent was obtained from patients included in the study.

\section{Author Contributions}

MG and GL conceptualized and designed the study, drafted the initial article, and reviewed and revised the article. The data were collected and analyzed by AM and SS. All authors approved the final article as submitted and agree to be accountable for all aspects of the work.

\section{Data Availability}

The datasets used and/or analyzed during the current study are available from the corresponding author on reasonable request.

\section{Abbreviations}

HLA-DR: human leukocyte antigen-DR isotype; mHLA-DR: HLA-DR expression on blood monocytes; PCT: procalcitonin; IFN: interferon; SOFA: Sequential Organ Failure Assessment; CPD: cell population data; NE: neutrophils; MO: monocytes; Ly: lymphocytes 


\section{References}

1. Medzhitov R. Origin and physiological roles of inflammation. Nature. 2008;454(7203):428-435.

2. Schulte W, Bernhagen J, Bucala R. Cytokines in sepsis: potent immunoregulators and potential therapeutic targetsan updated view. Mediators Inflamm. 2013;2013:165974.

3. Gentile LF, Cuenca AG, Efron PA, Ang D, Bihorac A, McKinley BA, Moldawer LL, et al. Persistent inflammation and immunosuppression: a common syndrome and new horizon for surgical intensive care. J Trauma Acute Care Surg. 2012;72(6):1491-1501.

4. Hotchkiss RS, Monneret G, Payen D. Sepsis-induced immunosuppression: from cellular dysfunctions to immunotherapy. Nat Rev Immunol. 2013;13(12):862-874.

5. Zorio V, Venet F, Delwarde B, Floccard B, Marcotte G, Textoris J, Monneret G, et al. Assessment of sepsis-induced immunosuppression at ICU discharge and 6 months after ICU discharge. Ann Intensive Care. 2017;7(1):80.

6. Arens C, Bajwa SA, Koch C, Siegler BH, Schneck E, Hecker A, Weiterer S, et al. Sepsis-induced long-term immune paralysis - results of a descriptive, explorative study. Crit Care. 2016;20:93.

7. Monneret G, Lepape A, Voirin N, Bohe J, Venet F, Debard AL, Thizy H, et al. Persisting low monocyte human leukocyte antigen-DR expression predicts mortality in septic shock. Intensive Care Med. 2006;32(8):1175-1183.

8. Martin MD, Badovinac VP, Griffith TS. CD4 T cell responses and the sepsis-induced immunoparalysis state. Front Immunol. 2020;11:1364.

9. Cao C, Yu M, Chai Y. Pathological alteration and therapeutic implications of sepsis-induced immune cell apoptosis. Cell Death Dis. 2019;10(10):782.

10. Luan YY, Yao YM, Xiao XZ, Sheng ZY. Insights into the apoptotic death of immune cells in sepsis. J Interferon Cytokine Res. 2015;35(1):17-22.

11. Jiang W, Zhong W, Deng Y, Chen C, Wang Q, Zhou M, Li X, et al. Evaluation of a combination "lymphocyte apoptosis model" to predict survival of sepsis patients in an intensive care unit. BMC Anesthesiol. 2018;18(1):89.

12. Hotchkiss RS, Nicholson DW. Apoptosis and caspases regulate death and inflammation in sepsis. Nat Rev Immunol. 2006;6(11):813-822.

13. Gupta DL, Bhoi S, Mohan T, Galwnkar S, Rao DN. Coexistence of Th1/Th2 and Th17/Treg imbalances in patients with post traumatic sepsis. Cytokine. 2016;88:214-221.

14. Boomer JS, To K, Chang KC, Takasu O, Osborne DF, Walton AH, Bricker TL, et al. Immunosuppression in patients who die of sepsis and multiple organ failure. JAMA. 2011;306(23):2594-2605.

15. Pachot A, Monneret G, Voirin N, Leissner P, Venet F, Bohe J, Payen D, et al. Longitudinal study of cytokine and immune transcription factor mRNA expression in septic shock. Clin Immunol. 2005;114(1):61-69.

16. Rimmele T, Payen D, Cantaluppi V, Marshall J, Gomez H, Gomez A, Murray P, et al. Immune Cell Phenotype and Function in Sepsis. Shock. 2016;45(3):282-291.

17. Monneret G, Venet F. Sepsis-induced immune altera- tions monitoring by flow cytometry as a promising tool for individualized therapy. Cytometry B Clin Cytom. 2016;90(4):376-386.

18. Singer M, Deutschman CS, Seymour CW, Shankar-Hari M, Annane D, Bauer M, Bellomo R, et al. The third international consensus definitions for sepsis and septic shock (Sepsis-3). JAMA. 2016;315(8):801-810.

19. Urrechaga E, Boveda O, Aguirre U. Improvement in detecting sepsis using leukocyte cell population data (CPD). Clin Chem Lab Med. 2019;57(6):918-926.

20. Greathouse KC, Hall MW. Critical illness-induced immune suppression: current state of the science. Am J Crit Care. 2016;25(1):85-92.

21. Manzoli TF, Troster EJ, Ferranti JF, Sales MM. Prolonged suppression of monocytic human leukocyte antigen-DR expression correlates with mortality in pediatric septic patients in a pediatric tertiary Intensive Care Unit. J Crit Care. 2016;33:84-89.

22. Greco M, Mazzei A, Palumbo C, Verri T, Lobreglio G. Flow cytometric analysis of monocytes polarization and reprogramming from inflammatory to immunosuppressive phase during sepsis. EJIFCC. 2019;30(4):371-384.

23. Zawada AM, Rogacev KS, Rotter B, Winter P, Marell RR, Fliser D, Heine GH. SuperSAGE evidence for CD14++CD16+ monocytes as a third monocyte subset. Blood. 2011;118(12):e50-61.

24. Funderburg NT, Zidar DA, Shive C, Lioi A, Mudd J, Musselwhite LW, Simon DI, et al. Shared monocyte subset phenotypes in HIV-1 infection and in uninfected subjects with acute coronary syndrome. Blood. 2012;120(23):45994608.

25. Chen P, Su B, Zhang T, Zhu X, Xia W, Fu Y, Zhao G, et al. Perturbations of Monocyte Subsets and Their Association with T Helper Cell Differentiation in Acute and Chronic HIV-1-Infected Patients. Front Immunol. 2017;8:272.

26. Hanna RN, Cekic C, Sag D, Tacke R, Thomas GD, Nowyhed H, Herrley E, et al. Patrolling monocytes control tumor metastasis to the lung. Science. 2015;350(6263):985990.

27. Yang J, Zhang L, Yu C, Yang XF, Wang H. Monocyte and macrophage differentiation: circulation inflammatory monocyte as biomarker for inflammatory diseases. Biomark Res. 2014;2(1):1.

28. Castano D, Garcia LF, Rojas M. Increased frequency and cell death of CD16+ monocytes with Mycobacterium tuberculosis infection. Tuberculosis (Edinb). 2011;91(5):348360.

29. Perez-Mazliah DE, Castro Eiro MD, Alvarez MG, Lococo B, Bertocchi G, Cesar G, Natale MA, et al. Distinct monocyte subset phenotypes in patients with different clinical forms of chronic Chagas disease and seronegative dilated cardiomyopathy. PLoS Negl Trop Dis. 2018;12(10):e0006887.

30. Passos S, Carvalho LP, Costa RS, Campos TM, Novais FO, Magalhaes A, Machado PR, et al. Intermediate monocytes contribute to pathologic immune response in Leishmania braziliensis infections. J Infect Dis. 2015;211(2):274-282.

31. Lin SJ, Lo M, Kuo RL, Shih SR, Ojcius DM, Lu J, Lee $\mathrm{CK}$, et al. The pathological effects of CCR2+ inflamma- 
tory monocytes are amplified by an IFNAR1-triggered chemokine feedback loop in highly pathogenic influenza infection. J Biomed Sci. 2014;21:99.

32. Shi C, Pamer EG. Monocyte recruitment during infection and inflammation. Nat Rev Immunol. 2011;11(11):762-774.

33. Greco M, Palumbo C, Sicuro F, Lobreglio G. Soluble Fmslike tyrosine kinase-1 is a marker of endothelial dysfunction during sepsis. J Clin Med Res. 2018;10(9):700-706.

34. Hotchkiss RS, Monneret G, Payen D. Immunosuppression in sepsis: a novel understanding of the disorder and a new therapeutic approach. Lancet Infect Dis. 2013;13(3):260268.

35. Hotchkiss RS, Tinsley KW, Swanson PE, Schmieg RE, Jr., Hui JJ, Chang KC, Osborne DF, et al. Sepsis-induced apoptosis causes progressive profound depletion of B and CD4+ T lymphocytes in humans. J Immunol. 2001;166(11):69526963.

36. Felmet KA, Hall MW, Clark RS, Jaffe R, Carcillo JA. Prolonged lymphopenia, lymphoid depletion, and hypoprol- actinemia in children with nosocomial sepsis and multiple organ failure. J Immunol. 2005;174(6):3765-3772.

37. Boomer JS, Shuherk-Shaffer J, Hotchkiss RS, Green JM. A prospective analysis of lymphocyte phenotype and function over the course of acute sepsis. Crit Care. 2012;16(3):R112.

38. Feuerecker M, Sudhoff L, Crucian B, Pagel JI, Sams C, Strewe C, Guo A, et al. Early immune anergy towards recall antigens and mitogens in patients at onset of septic shock. Sci Rep. 2018;8(1):1754.

39. Poujol F, Monneret G, Gallet-Gorius E, Pachot A, Textoris J, Venet F. Ex vivo stimulation of lymphocytes with IL-10 mimics sepsis-induced intrinsic T-cell alterations. Immunol Invest. 2018;47(2):154-168.

40. Washburn ML, Wang Z, Walton AH, Goedegebuure SP, Figueroa DJ, Van Horn S, Grossman J, et al. T cell- and monocyte-specific RNA-sequencing analysis in septic and nonseptic critically ill patients and in patients with cancer. J Immunol. 2019;203(7):1897-1908. 